\title{
Simple quasi-common path point diffraction interferometer with adjustable fringe contrast and carrier frequency
}

F. Z. Bai

fzbaiim@163.com

Y. Miao

H. Guo

hguo@suda.edu.cn

X. J. Gao

X. Q Wang
College of Mechanical Engineering, Inner Mongolia University of Technology, Huhhot 010051, China

College of Mechanical Engineering, Inner Mongolia University of Technology, Huhhot 010051, China

Robotics and Microsystems Center, Soochow University, Suzhou, 215021,China

College of Mechanical Engineering, Inner Mongolia University of Technology, Huhhot 010051, China

This paper presents a simple quasi-common path point diffraction interferometer (PDI) that allows fringe contrast and fringe spatial frequency to be adjusted conveniently. The novel aspect of this PDI is the use of a polarizer with pinhole as the PDI mask - and then, with a compact circular optical setup, reference and measurement waves are obtained. Furthermore, a linear tilting modulation is added into two interfering waves and adjusted easily by tilting the polarizing beam splitter - and hence the Fourier transform method can be perfectly applied to extract the wavefront phase from the captured fringe pattern, with the highest fringe contrast and suitable carrier frequency. Detailed theoretical analysis and experimental results are presented.

[DOI: http://dx.doi.org/10.2971/jeos.2015.15042]

Keywords: Phase measurement, point diffraction interferometer (PDI), fringe contrast, carrier frequency

\section{INTRODUCTION}

The point diffraction interferometer (PDI) is a self-referencing interferometer wavefront sensor (SRI WFS) with high spatial resolution, because each pixel corresponds to a sub-aperture in the interferogram. At the same time, this interferometric wavefront sensor based on PDI principles can carry out the absolute wavefront measurement without a requirement for precision reference optics and can directly obtain the wavefront information without the need for a reconstruction algorithm similar to that used in the shearing interfermoter [1, 2]. Also, it can be applied to detect the wavefront phase in strong scintillation $[2,3]$ and irregular pupil situations. Therefore, this wavefront sensor based on PDI principles is a very important component for improvement of the performance of adaptive optics (AO) systems. Various PDIs have been developed and applied in accurate phase measurement and fluid flow diagnostics since Smart et al. [4] proposed the PDI, in which the reference wave is generated by diffraction from a tiny pinhole and it has features like common-path and self-referencing.

The PDI with common-path structure is insensitive to vibration, has long coherence lengths and can minimize the influences of air turbulence or thermal fluctuation. However, traditional common path PDI also has some drawbacks in its difficulty of phase shifting [5] or wavefront extraction, poor realtime measurement and poor technology for making the PDI mask [6]-[8], as well as low fringe contrast $[1,4,5,9,10]$.

On the basis of the structure of the circular-path radial shear- ing interferometer [11]-[13] and the use of a polarizer with pinhole as the PDI mask, a circular quasi-common path PDI is proposed. The new PDI system allows fringe contrast and fringe spatial frequency to be simply adjusted, and then, by using the Fourier transform method (FTM), the wavefront phase is extracted in real-time. Compared with the traditional common path PDIs, the proposed PDI is compact in size and simple in configuration - as well as easily adjusted. Additionally, for the different wavefront phases under test, the fringe with the highest fringe contrast and suitable carrier frequency can be obtained in this PDI. Therefore, another potential advantage is that it can measure different wavefront phases with higher accuracy.

\section{PRINCIPLES OF THE PROPOSED PDI}

The arrangement of the proposed common path PDI is presented in Figure 1. A linearly polarized laser beam including the measured phase information passes through a polarizing beam splitter (PBS) and is divided into a reflected beam and a transmitted beam with orthogonally polarized orientations. At the same time, the amplitude proportion of the two optical beams is adjusted as the half-wave plate (HWP) placed in front of the PBS is rotated. Then the transmitted and the reflected beams traverse into a circular telescope system composed of two reflecting mirrors (M1 and M2) and two lenses (L1 and L2) with equivalent focal lengths. These two beams travel along nearly the same optical path but along opposite directions - namely, the transmitted beam traverses along 


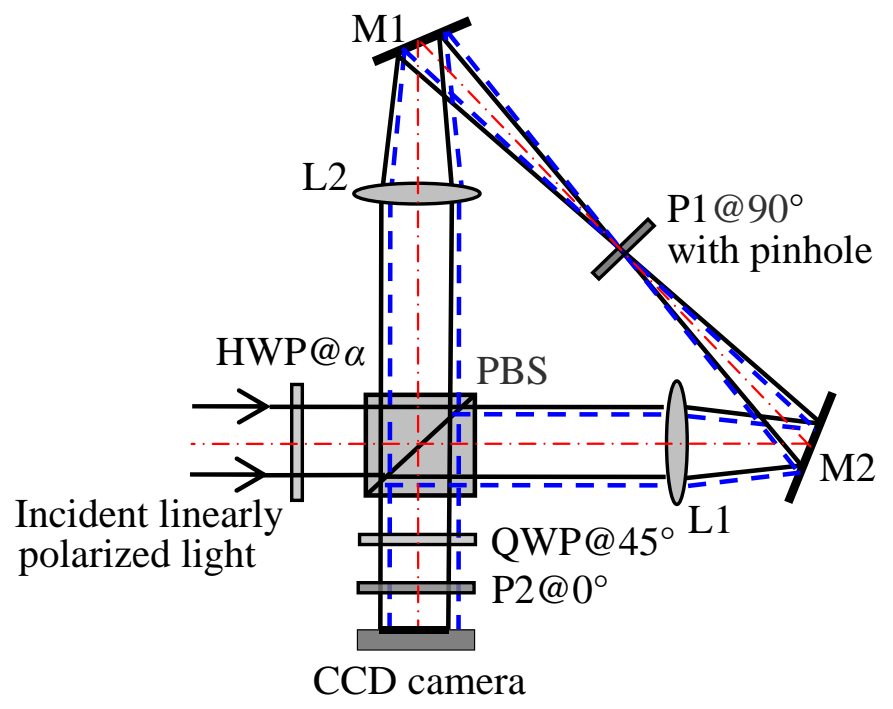

FIG. 1 Schematic diagram of quasi-common path PDI. HWP, half-wave plate; PBS, polarization beam splitter; L1-2, lenses; M1-2, mirrors; QWP, quarter-wave plate; P, polarier.

the anti-clockwise direction and the reflected beam traverses along the clockwise direction, which are represented as the solid line and the dotted line in Figure 1, respectively. A polarizer (P1) with a pinhole as the PDI mask is placed in the focal plane of L1 and L2 and the transmission axis of P1 is perpendicular to the polarization direction of the transmitted beam but parallel to that of the reflected beam. Therefore, the transmitted beam focused on the pinhole in the P1 will produce diffraction and makes a reference wave afterwards, whereas the reflected beam will be focused outside of the pinhole (i.e., focused on the P1) while the PBS is tilted. Thus the reflected beam passes through the P1 with almost no attenuation and makes a test wave. Then, once again, the reference and the test waves will be wholly transmitted and reflected from the PBS, respectively. The interference fringe pattern between the reference and test waves is captured by a CCD camera after a quarter-wave plate (QWP) with its fast axis at $45^{\circ}$ and a polarizer (P2) for qualitative analysis. Each wave is represented by a Jones matrix for mathematical interpretation. The incident linearly polarized light expressed as $E=\exp (i \phi)$ is divided into two orthogonal beams: the transmitted beam $\cos \alpha\left[\begin{array}{l}1 \\ 0\end{array}\right] \exp (i \phi)$ and the reflected beam $\sin \alpha\left[\begin{array}{l}0 \\ 1\end{array}\right] \exp (i \phi)$, where $i=\sqrt{-1}, \phi$ is the wavefront phase to be measured, and $\alpha$ ranging from 0 to $90^{\circ}$ is the fast axis direction of the HWP. The Jones matrix of the P1 with its transmission axis at $90^{\circ}$ is $\left[\begin{array}{ll}0 & 0 \\ 0 & 1\end{array}\right]$. It is assumed that the phase filtering after pinhole diffraction is perfect, so the transmitted beam will become a plane wave and is then regarded as the reference wave, which is expressed as:

$$
E_{r}=\sqrt{T} \cos \alpha\left[\begin{array}{l}
1 \\
0
\end{array}\right]
$$

namely the phase information included in the factor $\exp (i \phi)$ in the above expression is eliminated. By pinhole filtering, the optical intensity passing through the pinhole will reduced and the parameter $T$ in Eq. (1) denotes the intensity transmission of the pinhole under a given wavefront aberration $\phi$. The undiffracted test wave from the reflected beam will hold the same amplitude and polarization direction with respect to the reflected beam and its Jones matrix is given by

$$
E_{m}=\left[\begin{array}{ll}
0 & 0 \\
0 & 1
\end{array}\right] \sin \alpha\left[\begin{array}{l}
0 \\
1
\end{array}\right] \exp (i \phi)=\sin \alpha\left[\begin{array}{l}
0 \\
1
\end{array}\right] \exp (i \phi)
$$

The reference and the test waves become right-circularly and left-circularly polarized after the QWP with its fast axis at $45^{\circ}$ for which the Jones matrices are $\frac{1}{\sqrt{2}}\left[\begin{array}{cc}1 & -i \\ -i & 1\end{array}\right]$, and are expressed respectively as

$$
\begin{aligned}
E_{r}^{\prime} & =\frac{1}{\sqrt{2}}\left[\begin{array}{cc}
1 & -i \\
-i & 1
\end{array}\right] \sqrt{T} \cos \alpha\left[\begin{array}{l}
1 \\
0
\end{array}\right] \\
& =\frac{\sqrt{T} \cos \alpha}{\sqrt{2}}\left[\begin{array}{c}
\exp (i 0) \\
\exp (-i \pi / 2)
\end{array}\right] \\
E_{m}^{\prime} & =\frac{1}{\sqrt{2}}\left[\begin{array}{cc}
1 & -i \\
-i & 1
\end{array}\right] \sin \alpha\left[\begin{array}{l}
0 \\
1
\end{array}\right] \exp (i \phi) \\
& =\frac{\sin \alpha}{\sqrt{2}}\left[\begin{array}{c}
\exp (-i \pi / 2) \\
\exp (i 0)
\end{array}\right] \exp (i \phi)
\end{aligned}
$$

Then two circularly polarized waves are recombined as follows,

$$
\left[\begin{array}{c}
E_{x} \\
E_{y}
\end{array}\right]=\frac{1}{\sqrt{2}}\left[\begin{array}{c}
\sqrt{T} \cos \alpha+\sin \alpha \exp [i(\phi-\pi / 2)] \\
\sqrt{T} \cos \alpha \exp (-i \pi / 2)+\sin \alpha \exp (i \phi)
\end{array}\right]
$$

The recombined wave passes through P2 with its transmission axis at $0^{\circ}$ for which the Jones matrix is $\left[\begin{array}{ll}1 & 0 \\ 0 & 0\end{array}\right]$, so only the component in the $\mathrm{x}$-direction is passed and the following expression is obtained:

$$
E_{x}=\frac{\sqrt{T} \cos \alpha}{\sqrt{2}}+\frac{\sin \alpha}{\sqrt{2}} \exp [i(\phi-\pi / 2)]
$$

So the intensity of the interferogram is expressed as:

$$
I=E_{x} E_{x}^{*}=\frac{T \cos ^{2} \alpha}{2}+\frac{\sin ^{2} \alpha}{2}+\sqrt{T} \cos \alpha \sin \alpha \sin \phi
$$

Assume that $I_{r}=\frac{T \cos ^{2} \alpha}{2}$ and $I_{m}=\frac{\sin ^{2} \alpha}{2}$, Eq. (7) is rewritten as:

$$
I=I_{r}+I_{m}+2 \sqrt{I_{r} I_{m}} \sin \phi
$$

When the PBS is rotated slightly, a linear tilting modulation is introduced between the two interfering waves. In this case, the interference fringe pattern can generally be described as:

$$
I=I_{r}+I_{m}+2 \sqrt{I_{r} I_{m}} \sin \left(\phi+2 \pi f_{0} x\right)
$$

where $f_{0}$ is the spatial carrier frequency introduced in the $\mathrm{x}$ direction. By using the FTM, the wavefront phase can be extracted from the fringe pattern, as shown in Eq. (9).

\section{THE PINHOLE DIAMETER AND FRINGE CONTRAST}

According to Fourier optics theory, the influence of the pinhole size on the precision and transmission intensity of the reference wave can be analyzed. Detailed analysis on the derivation of the reference wave in the PDI is given in our previous work reported in the literature $[14,15]$. Here, only two important conclusions are presented. We use 3 to 25 Zernike modes to describe the original wavefront phase, and their 


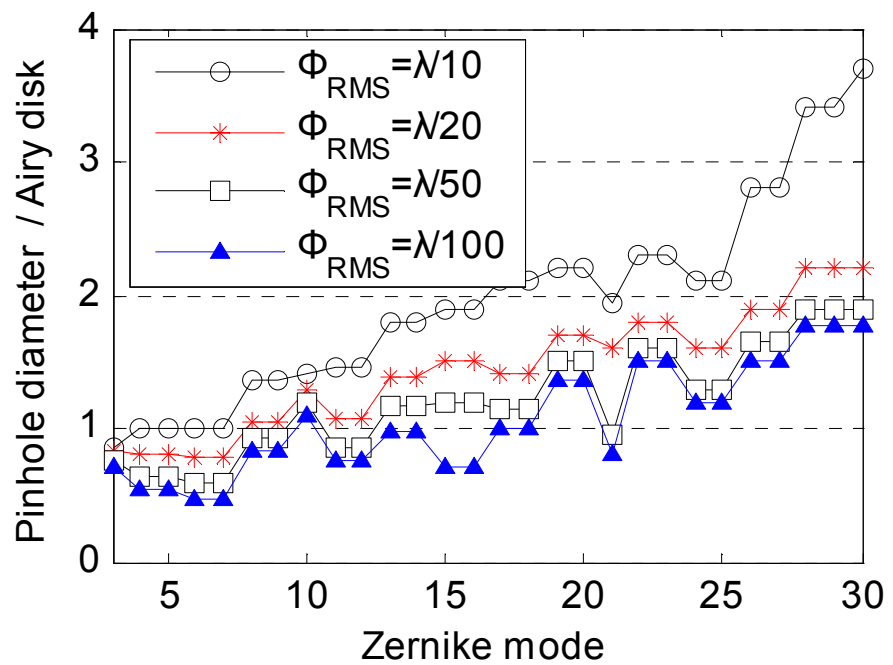

FIG. 2 Pinhole size required for different Zernike modes to produce the reference phase with given RMS value.

peak-to-valley (PV) values are normalized withs respect to wavelength. These different wavefront aberrations are filtered by the pinhole - and then the root-mean-square (RMS) value of the reference phase is calculated and is described as $\Phi_{\text {RMS }}$. For different Zernike modes to produce the reference wavefront with a given $\Phi_{\mathrm{RMS}}$, the required pinhole diameter is shown in Figure 2. The simulated results show that, for all Zernike modes, to obtain a higher accuracy reference phase a bigger pinhole is needed. Furthermore, in the case of the same $\Phi_{\mathrm{RMS}}$, the pinhole sizes required for low-order Zernike modes are generally less than those required for high-order modes. Therefore, for optical testing, the requirement of a high accuracy reference wave suggests that the pinhole should be not greater than half of the Airy disk for the unaberrated wave. In this case, the accuracy of the reference phase will reach $\lambda / 100$. At the same time, for certain Zernike aberrations, the accuracy may even reach $\lambda / 10^{4}$.

The pinhole in the PDI structure not only produces a reference phase that is near to a plane wave but also reduces the transmission intensity. An unaberrated plane wave with unity amplitude is taken into account. It can be concluded from Figure 3 that when the pinhole diameter equals half of the Airy disk the intensity transmission equals 63\%. Moreover, a smaller pinhole will restrict more light energy to be passed. In addition, it is obvious from Figure 4 that, for an aberrated wavefront, the transmission intensity will decrease further and the transmission intensity is completely different for every Zernike mode. Therefore, to improve the fringe visibility we need a significant time to adjust the intensity of test wave. However, that is not a practicable scheme in common path systems.

The fringe contrast is one of the important factors affecting the accuracy of an interferometer [16]. According to the intensity formula of the interferogram shown as Eq. (9), the fringe contrast is given by

$$
K=\frac{2 \sqrt{I_{r} I_{m}}}{I_{r}+I_{m}}=\frac{2 T \cos \alpha \sin \alpha}{T \cos ^{2} \alpha+\sin ^{2} \alpha}=\frac{2 \sqrt{T} \tan \alpha}{T+\tan ^{2} \alpha}
$$

If we let $K$ in Eq. (10) be equal to one, then the relationship

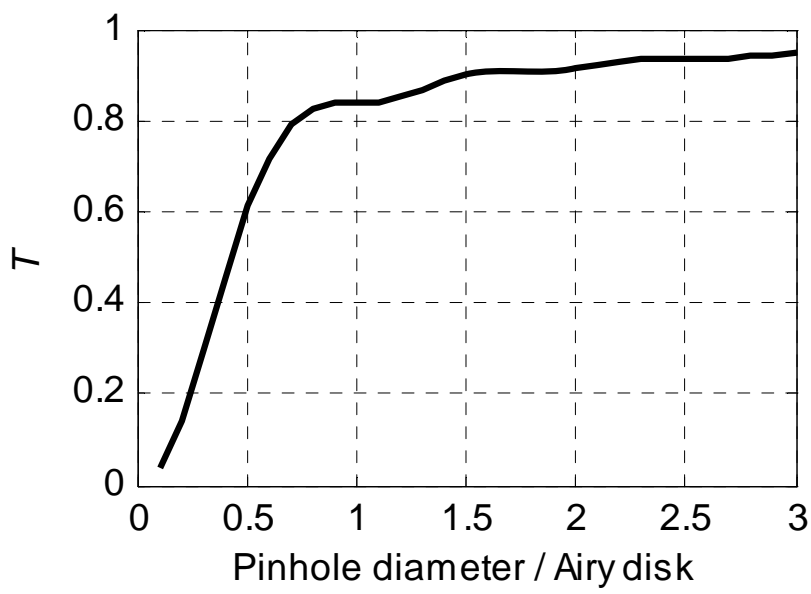

FIG. 3 Intensity transmission of reference wave versus increasing pinhole size.

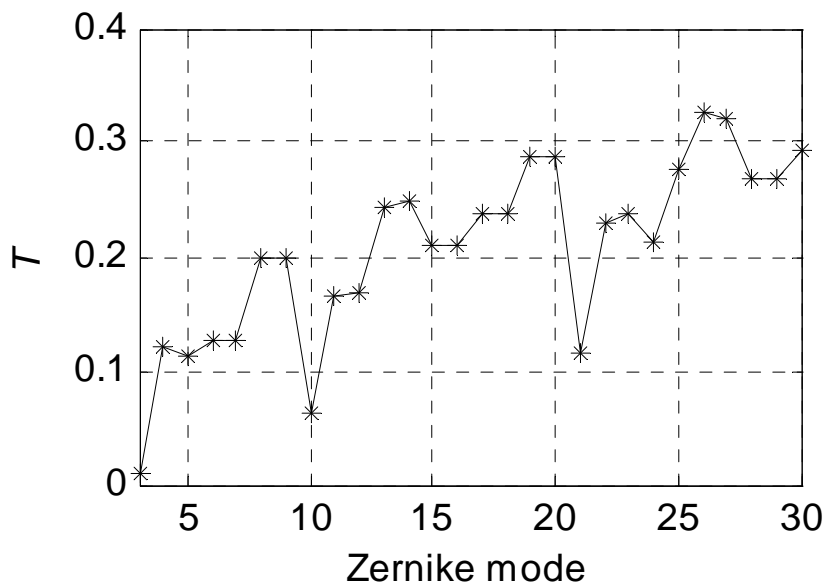

FIC. 4 Intensity transmission of different Zernike modes traveling through a pinhole with half of the Airy disk.

between parameters $\alpha$ and $T$ is described as

$$
\alpha=\arctan (\sqrt{T})
$$

From Eq. (10) it can be deduced that the rotation angle $\alpha$ of the HWP influences the fringe contrast $K$, namely, the fringe contrast in the proposed PDI can be adjusted by rotating the HWP. At the same time, Eq. (11) indicates that, in the case of a given transmission intensity, T one can obtain a value of $\alpha$ that makes the fringe contrast of the interferometer reach its highest value (i.e. one). Furthermore, it has been shown in the above analysis that the transmission intensity is influenced by two factors, i.e. the pinhole size and the measured wavefront phase. Therefore, the transmission intensity $T$ will vary, along with the measured wavefront phase $\phi$. In this case, the maximal contrast $K$ can be obtained by adjusting the angle $\alpha$.

Here, we use an atmospheric phase screen consistent with Kolmogorov theory to simulate the situation numerically. The phase screen with $D / r_{0}=5$ consists of 3 to 25 Zernike modes, where $D$ is the input aperture diameter and $r_{0}$ is the Fried parameter, and a wrapped phase is shown in Figure 5(a). A pinhole with $60 \%$ of the Airy disk diameter for the unaberrated wave is used to generate the reference wave and moderately improve the transmission intensity. In the case of a given pinhole size and an aberrated phase as shown in Figure 5(a), the fringe contrast $K$ as a function of the rotation angle $\alpha$ of the HWP is illustrated in Figure 5(b). 


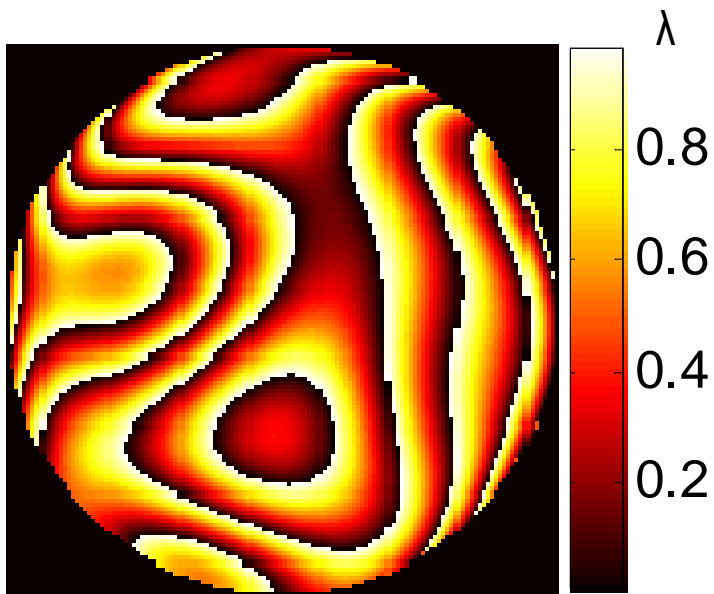

(a)

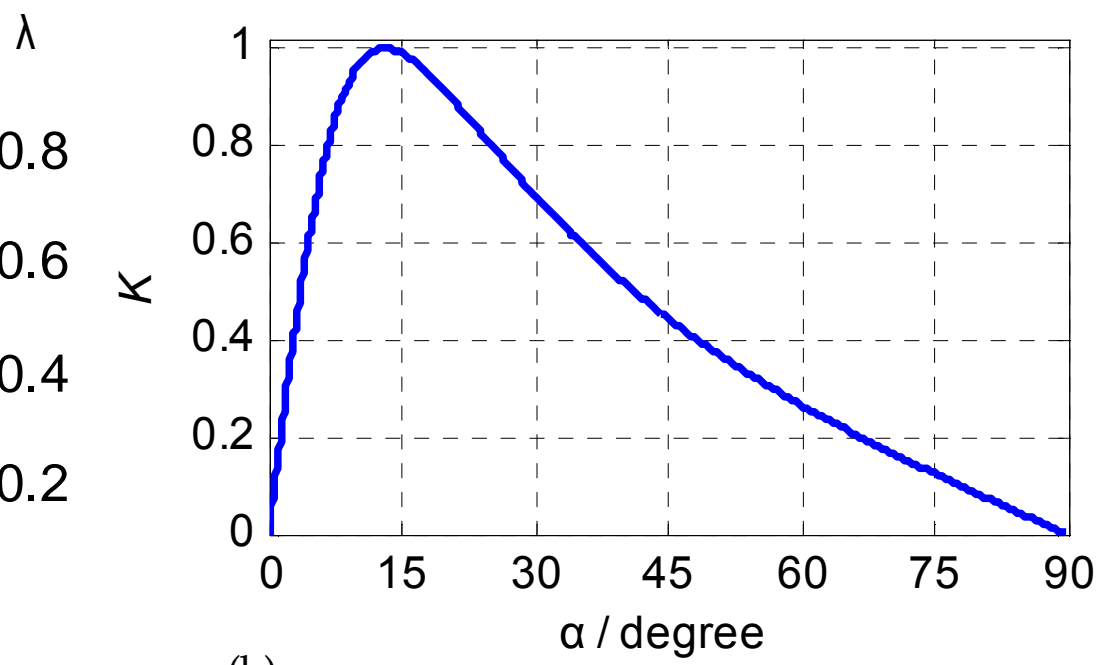

(b)

FIG. 5 Simulated results of fringe contrast. (a) wrapped atmospheric phase screen with $D / r_{0}=5$, (b) fringe contrast versus rotation angle of the HWP under the phase shown in (a).

From Figure 5(b) it can be deduced that, by varying $\alpha$, the intensity ratio of the two interfering beams can be adjusted in order to maximize the fringe contrast, which can reach unity in the case of any an input wavefront aberration. As a result, in the proposed common path PDI, the fringe contrast can be adjusted easily - whereas this is very difficult in many previous common path PDI systems $[1,4,5,9,10]$.

\section{EFFECT OF CARRIER FREQUENCY TO THE FTM}

The FTM was originally introduced and demonstrated by Takeda et al $[17,18]$, and only one frame fringe pattern is required, which makes it the easiest method capable of dealing with dynamic situations. To descript briefly the principle of the FTM, generally, the carrier frequency fringe shown as Eq. (9) can be rewritten as

$$
g(x, y)=a(x, y)=b(x, y) \cos \left[\phi(x, y)=2 \pi f_{0} x\right],
$$

where $a(x, y)$ and $b(x, y)$ are the background and the modulation intensity, respectively. Assuming that $a(x, y), b(x, y)$ and $\phi(x, y)$ are slowly varying functions compared with the spatial carrier frequency $f_{0}$, that is

$$
f_{0}>\frac{\delta a(x, y)}{\delta x}, \frac{\delta b(x, y)}{\delta x}, \text { and } \frac{\delta \phi(x, y)}{\delta x}
$$

In this case, the three terms in the Fourier transforms of Eq. (12) will be separable. Thus a suitable frequency filter is used to isolate any single fundamental frequency composition. Then the phase $\phi(x, y)$ can be retrieved with the FTM. Figure 6 shows a frame of simulated fringe patterns with $f_{0}=25$. Through variation of the carrier frequency, a series of fringe patterns can been generated. With the use of FTM, the phases of all the simulated fringes are extracted. The difference between the extracted and the given phases is obtained and defined as the residual phase. Also, the RMS values of the residual phases are calculated. So the behavior of the RMS value versus the carrier frequency under the given aberration is plotted in Figure 7.

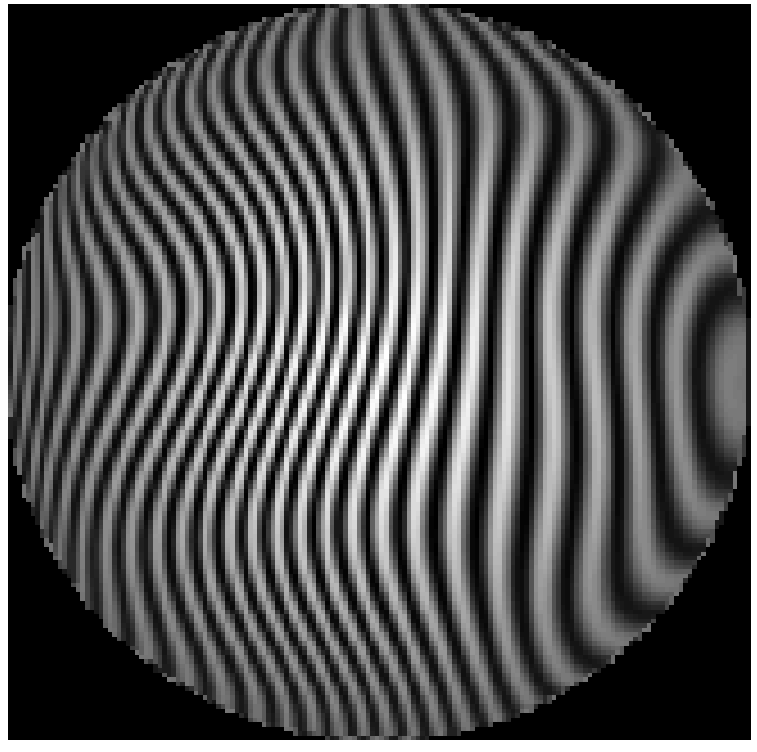

FIG. 6 Simulated fringe pattern with $f_{0}=25$.

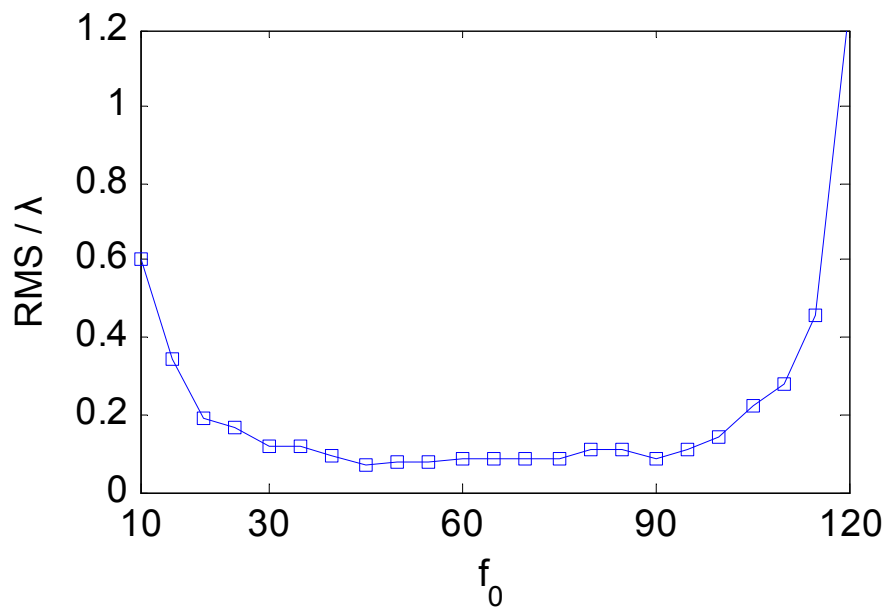

FIC. 7 RMS value of residual phase versus carrier frequency under a series of simulated fringe such as Figure 6.

The result from Figure 7 reflects the affecting law of carrier frequency to the FTM, namely, a suitable carrier frequency is of great importance for the FTM especially while the practical aberration to be measured is unknown, which holds widerange alterability such as different aberration modes or vary- 

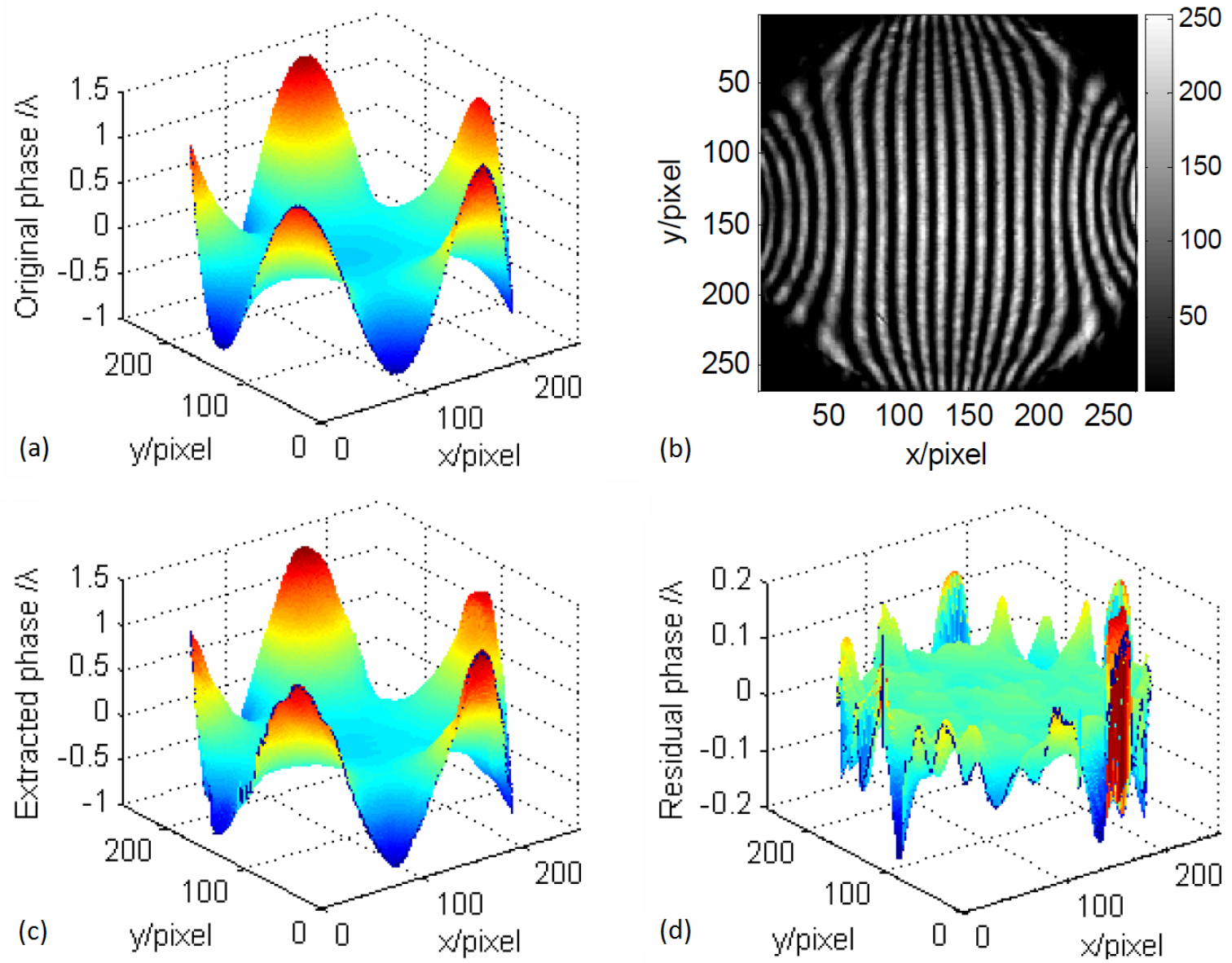

FIG. 8 Experimental results. (a) original phase introduced by the SLM, (b) fringe pattern captured by the proposed PDI, (c) wavefront phase extracted from (b), (d) residual phase error.

ing magnitude. In fact, in the case of total separation of the zero-frequency and fundamental-frequency compositions, the lower carrier frequency may increase the spatial sampling resolution to one fringe period. As a result, a higher phase extraction accuracy can be obtained.

However, the determination of the carrier frequency depends mainly on the varying speed of $a(x, y), b(x, y)$ and $\phi(x, y)$ in Eq. (12). Therefore, an interferometer system with adjustable carrier frequency is very helpful in improving the accuracy of the FTM to the maximum. This characteristic is very useful, especially in the case where the PDI is used in a closed-loop AO system. In the beginning of closed-loop correction, the spatial variation of the incident wavefront is faster. In this case, the higher carrier frequency may increase the accuracy of phase measurement, accelerating the closed-loop correction speed. When the correction process is close to completion, the residual wavefront is slowly varying. In this case, the lower carrier frequency may increase the number of samples acquired for each fringe period, improving the precision of the final correction result. As a result, the adjustable carrier frequency can be achieved easily in the proposed quasi-common path PDI whereas it was very difficult in some previous common path PDIs $[1,10]$.

\section{EXPERIMENTAL RESULTS}

In the experimental system, the collimated-plane laser output is reflected from a reflective liquid-crystal spatial light modulator (SLM) and then is incident into the PDI system, as shown in Figure 1. The SLM that works in phase-only modulation mode is used to introduce the aberrated wavefront to be tested. The SLM used has an array of $256 \times 256$ pixels across a $4.61 \mathrm{~mm}$ square aperture and the relationship between the phase retardance and the gray levels has been calibrated accurately in our previous work [19]. In the PDI, a $60 \mu \mathrm{m}$ pinhole is used, which equals about $60 \%$ of the Airy disk for unaberrated wave, namely $1.46 \lambda f / D$, where $f(250 \mathrm{~mm})$ is the focal length of L1 and L2 in Figure 1, $\lambda(632.8 \mathrm{~nm})$ is the laser wavelength, and $D(4.61 \mathrm{~mm})$ is the aperture size.

At first, an aberrated wavefront is produced by the SLM with a phase wrapping operation (modulo $2 \pi$ ). Then it is measured by a Zygos interferometer with a vibration-isolating platform and the measured result, as shown in Figure 8(a), is also used as the original phase distribution. While this aberrated wavefront is introduced, the corresponding fringe pattern is captured by the CCD camera in the PDI. Next, by rotating gradually the HWP a series of fringe patterns with different contrast are captured and their corresponding contrast values are calculated. According to the fitting results, the rotation angle of the HWP associated with the highest contrast is determined - and then, by rotating the HWP and tilting the PBS, a fringe pattern with the highest fringe contrast and a suitable carrier frequency is captured and shown in Figure 8(b). Finally, By using the FTM the wavefront phase is extracted from Figure 8(b) and is shown in Figure $8(\mathrm{c})$. The residual phase, i.e., the difference between the extracted and the original phases, is calculated and also 
shown in Figure 8(d). The RMS value and the peak to valley value for the residual phase shown in Figure 8(d) are equal to $0.03 \lambda$ and $0.44 \lambda$ respectively. It can been seen from Figure $8(d)$ that the error of the inner area in the extracted result is very low, except for the border part of the analyzed fringe pattern. The maximal errors that appear in the area near to the borders are mainly due to the well-known border effect [20] from the FTM. This problem can be overcome using techniques such as the published literature [21] for improving the accuracy of phase extraction, especially in the border part.

The experimental procedure illustrates that this simple and compact PDI system is convenient to set up and use. Also, the experimental results show that the proposed quasi-common path PDI can realize the adjustment of fringe contrast and fringe carrier frequency, and hence higher accuracy phase extraction is obtained. Moreover, it is known that the fringe contrast is directly related to the parameter of intensity transmission. When this parameter is obtained, the accurate rotation angle of the HWP can be known. In this case, automatic rotation to the HWP may realize the maximization of fringe contrast so as to reach real-time measurement in the future.

\section{CONCLUSIONS}

This paper has presented a new quasi-common path PDI to extract the wavefront phase from a carrier frequency fringe pattern. Detailed analysis of principles and experimental work has been carried out - and it is shown that the fringe contrast and the carrier frequency can be adjusted easily and hence one can obtain a fringe pattern with the highest fringe contrast and suitable carrier frequency when this PDI operates. Another main virtue of this PDI is that it is simple to set up and use, compact in structure, has easy fabrication of the PDI mask, is flexible to use, and is a low cost system. The PDI proposed in the paper is also of great practicality as an SRI WFS using closed-loop AO, because the fringe contrast and the carrier frequency need to be adjusted as the input phase varies gradually.

\section{ACKNOWLEDGMENTS}

This work was supported by the National Natural Science Foundation of China under grant No. 61108038, and the Natural Science Foundation of Inner Mongolia of China under grant No. 2011BS0701 and 2015MS0616.

\section{References}

[1] H. Medecki, E. Tejnil, K. A. Goldberg, and J. Bokor, "Phase-shifting point diffraction interferometer," Opt. Lett. 21, 1526-1528 (1996).

[2] J. D. Barchers, D. L. Fried, D. J. Link, G. A. Tyler, W. Moretti, T. J. Brennan, and R. Q.Fugate, "The performance of wavefront sensors in strong scintillation," Proc. SPIE. 4839, 217-227 (2003)

[3] J. Notaras, and C. Paterson, "Point-diffraction interferometer for atmospheric adaptive optics in strong scintillation," Opt. Commun. 281, 360-367 (2008).
[4] R.N. Smartt, and W.H. Steel, "Theory and application of pointdiffraction interferometers," Jpn. J. Appl. Phys. 14, 351-356 (1975).

[5] H. Kihm, and Y. W. Lee, "Double-pass point diffraction interferometer," Meas. Sci. Technol. 21, 105307 (2010).

[6] C. R. Mercer, and K. Creath, "Liquid-crystal point-diffraction interferometer for wave-front measurements," Appl. Optics 35, 1633-1642 (1996).

[7] C. R. Mercer, and K. Creath, "Liquid-crystal point-diffraction interferometer," Opt. Lett. 19, 916-918 (1994).

[8] M. J. Guardalben, and N. Jain, "Phase-shift error as a result of molecular alignment distortions in a liquid-crystal point- diffraction interferometer," Opt. Lett. 25, 1171-1173 (2000).

[9] M. Paturzo, F. Pignatiello, S. Grilli, S. D. Nicola, and P. Ferraro, "Phase-shifting point-diffraction interferometer developed by using the electro-optic effect in ferroelectric crystals," Opt. Lett. 31, 3597-3599 (2006).

[10] H. Furuhashi, A. Shibata, Y. Uchida, K. Matsuda, and C. P. Grover, "A point diffraction interferometer with random-dot filter," Opt. Commun. 237, 17-24 (2004).

[11] M. V. R. K. Murty, "A compact radial shearing interferometer based on the law of refraction," Appl. Optics 3, 853-857 (1964).

[12] N. T. Gu, L. H. Huang, Z. P. Yang, and C. H. Rao, “A single-shot common-path phase-stepping radial shearing interferometer for wavefront measurements," Opt. Express 19, 4703-4713 (2011).

[13] Y. Liu, F. Z. Bai, Y. Q. Wu, S. M. Gan, Z. Liu, and X. Y.Bao, “A common-path radial shearing phase-shifting interferometer with adjustable fringe contrast," Acta Optica Sinica 33, 0622003 (2013).

[14] F. Z. Bai, X. Q. Wang, K. Z. Huang, N. T. Gu, S. M. Gan, and F. Tian, "Analysis of spatial resolution and pinhole size for single-shot point-diffraction interferometer using in closed-loop adaptive optics," Opt. Commun. 297, 27-31 (2013).

[15] F. Z. Bai, and C. H.Rao, "Effect of pinhole diameter on correction accuracy of closed-loop adaptive optics system using selfreferencing interferometer wavefront sensor," Acta Phys. Sin- $\mathrm{CH}$ ED 59, 8280-8286 (2010)

[16] D. D. Wang, Y. Y. Yang, C. Chen, and Y. M. Zhuo, "Point diffraction interferometer with adjustable fringe contrast for testing spherical surfaces," Appl. Optics 50, 2342-2348 (2011).

[17] M. Takeda, H. Ina, and S. Koboyashi, "Fourier transform methods of fringe-pattern analysis for computer-based topography and interferometry," J. Opt. Soc. Am. 72, 156-160 (1982)

[18] M. Takeda, and K. Mutoh, "Fourier transform profilometry for the automatic measurement 3-D object shapes," Appl. Optics 22, 3977-3982 (1983).

[19] F. Z. Bai, and C. H. Rao, "Experimental validation of closed-loop adaptive optics based on a self-referencing interferometer wavefront sensor and a liquid-crystal spatial light modulator," opt. Commun. 283, 2782-2786 (2010).

[20] R. J. Green, J. G. Walker, and D. W. Robinson, "Investigation of the Fourier-transform method of fringe pattern analysis," Opt. Lasers Eng. 8, 29-44 (1988).

[21] M. Kujawinska, and J. Wojciak, "High accuracy Fourier transform fringe pattern analysis," Opt. Lasers Eng. 14, 325-339 (1991). 